\title{
Influence of the ethylene glycol, water treatment and microwave irradiation on the characteristics and performance of VPO catalysts for n-butane oxidation to maleic anhydride
}

\begin{abstract}
In this paper, the preparation of vanadium phosphate catalysts was shown to be improved by (1) using V2O5 and ethylene glycol as starting and reducing agent material, respectively for VOPO4 - 2H2O, (2) subsequent water treatment and (3) microwave irradiation. In particular, the preparation route, based on the reduction of VOPO $4 \cdot 2 \mathrm{H} 2 \mathrm{O}$ with various alcohols, is described in detail and contrasted with other three established methods performed by using ethylene glycol and isobutyl alcohol as reductant and solvent for V2O5 or distilled water as a solvent material. The preparation of catalyst precursor is carried out by two different methods, namely conventional heating and microwave irradiation. With this new technique, catalysts derived from the reduction of VOPO4 . $2 \mathrm{H} 2 \mathrm{O}$ by ethylene glycol exhibit substantially higher surface area (typically $>40 \mathrm{~m} 2 \mathrm{gl} 1$ ) and activity. In fact, the surface area of the catalyst is significantly enhanced when the precursor is refluxed by distilled water and dried by microwave heating. The characterization of catalysts was carried out using X-ray diffraction (XRD), Brunauerï Emmerï Teller (BET) surface area measurement, temperature programmed reduction (H2-TPR), temperature-programmed reaction (TPRn) and scanning electron microscopy (SEM). This study shows that employing ethylene glycol as reducing agent, followed by adding the water treatment step to catalyst synthesis procedure, and using microwave irradiation would give rise to enhanced surface area, activity and selectivity of the catalyst. Moreover, it introduces a more energy efficient procedure for preparation of vanadium phosphate catalyst used in selective oxidation of n-butane process.
\end{abstract}

Keyword: Vanadium phosphate catalyst; Ethylene glycol; Water treatment; Microwave irradiation; TPRn; n-butane oxidation 